\title{
Influence of Orthodontist's Gender and Experience on the Perception of Dentoskeletal Parameters Using Cephalometric Radiographs: A Questionnaire Study
}

\author{
Eslam R. Elkordy ${ }^{1 *}$, Essam M. Nasef ${ }^{1,2}$, Fouad A. El Sharaby ${ }^{2}$ \\ ${ }^{1}$ Department of Orthodontics and Dentofacial Orthopedics, Faculty of Dentistry, Future University in Egypt, Cairo, Egypt; \\ ${ }^{2}$ Department of Orthodontics and Dentofacial Orthopedics, Faculty of Dentistry, Cairo University, Cairo, Egypt
}

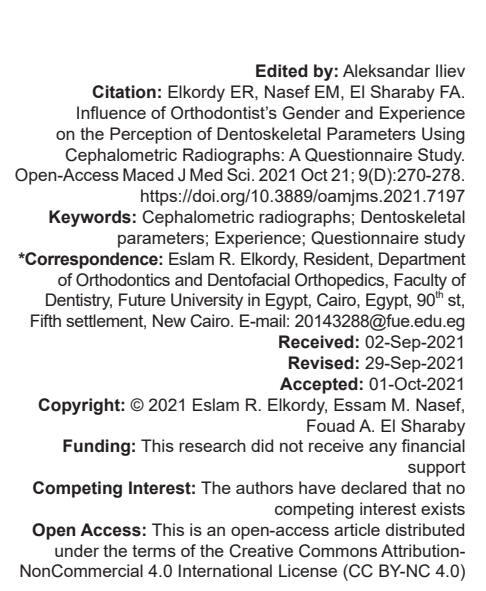

\section{Abstract}

AIM: The aim of this study was to evaluate the influence of orthodontist's gender and experience on the perception of dentoskeletal parameters through cephalometric radiographs.

METHODS: An online survey was developed using six laterals cephalometric radiographs. The survey included questions about clinicians' demographics as well as questions about the selected radiographs. The survey was distributed through Egyptian association of orthodontist's partner to 200 members.

RESULTS: Comparison between orthodontists with three levels of experience showed that there was a statistically significant difference between the three groups regarding total score of conformity of eyeball tracing with digital tracing results $(p=0.004$, effect size $=0.085)$. Pair-wise comparisons revealed that orthodontists with more than 10 years of experience showed the statistically significantly highest median score. There was no statistically significant difference between orthodontists with experience $<5$ years and those with $5-10$ years of experience; both showed statistically significantly lower median scores.

CONCLUSION: Orthodontists with more than 10 years of experience showed higher prevalence of perception of dentoskeletal parameters on lateral cephalometric radiographs than the less experienced groups. No association was found between clinician's gender and perception of dentoskeletal parameters.

\section{Introduction}

Successful orthodontic treatment begins with the correct diagnosis. Many different factors play a role in the treatment planning process and they vary greatly from one patient to another and from one orthodontist to another. Clinicians' gender and experiences are perceived as central factors that play a substantial role in diagnosis and treatment planning [1]. The significance of clinician's experience becomes apparent when different orthodontists are given the same patient scenario to evaluate. While different philosophies have developed over the years, the reason behind the inconsistencies in diagnosis for borderline cases is still unclear [2].

Cephalometric radiography analysis is considered to be part of the "gold" standard for diagnosis at the start of orthodontic treatment together with dental casts, intra- and extra-oral photographs, and panoramic radiographs [3].

Literature on what orthodontist characteristics may affect case perception, diagnosis, and treatment decisions is limited. The few studies that have looked at which clinician's traits influence the treatment decision were usually done as part of larger studies focusing on patient differences. The data from these few studies were conflicting. Some have discovered an association between clinicians' gender and experience with extraction treatment decisions while others did not find any relationship between the two. Some has attributed the differences among clinicians to either judgmental or perceptual variations [1].

It was reported that perceptual differences result from two people interpreting the same thing differently while judgmental variations were seen when two people agree on what they see, but disagree on the treatment. Clinician's past experiences can influence both the perceptual and the judgmental aspects of the decision-making process. The influence of clinician's educational background and philosophy, gender, and level of experience in diagnosis and treatment planning cannot be ignored. There is a paucity of data in literature concerning influence of orthodontist's gender and experience on the perception of dentoskeletal parameters. Therefore, this study was conducted to investigate this issue. 
The null hypothesis for the current study was that there is no difference between orthodontists of different level of experience as far as perception of dentoskeletal parameter on lateral cephalometric radiographs.

\section{Materials and Methods}

The study was performed in the outpatient clinic of the Orthodontic Department in the Faculty of Oral and Dental medicine of the Future University in Egypt. Fifty lateral cephalometric radiographs were collected randomly from Orthodontic Department Future University in Egypt and stored in computer database then imported to the digital cephalometric analysis software (AudaxCeph advantage, 4.1.2.3052). Before digital tracing of landmarks with AudaxCeph, the films were calibrated by digitizing two points on the ruler within the digital cassette. Variables were automatically generated by the program once a set of landmarks have been digitized. Digital tracings and measurements were performed by single orthodontist 4 times and the mean of reading of the 4 times for each radiograph was considered as golden standard for that case table (Tables 1-6). The golden standard tracing of the cases was given score [1], while incorrect tracing was recorded as score [0]. If the orthodontist's eyeballs tracing was the same as golden standard score, so he/she was given score 1 and if it was incorrect, he/she was given score 0 . The collected radiographs were categorized into three groups, Group 1: Included records of patients with Class I dentoskeletal relation, Group 2: Included records of patients with Class II dentoskeletal relation, and Group 3: Included records of patients with Class III dentoskeletal relation.

Out of 50 collected radiographs, six lateral radiographs were selected with the best patient orientation, exposure, and printing quality. Each group included two lateral cephalometric radiographs, one with horizontal growth pattern and the second with vertical growth pattern (Figures 1-6)

These radiographs were chosen by the coauthor of this study. Inclusion criteria for radiographs were as follows: Orthodontic patients (from 10 to 30 years old), orthodontic patients with skeletal Class I, II, and III and good quality radiographs without any defect, radiographs with correct head position, good contrast, magnification ratio $1: 1$, and patient biting in occlusion (maximum intercuspation).

The electronic survey was developed using SurveyMonkey ${ }^{\circledR}$. The questionnaire was consisted of two sections. Section one included demographic data related to the participant orthodontists including years of experience, gender, and place of education. The second section included patient's records (six cases) to be evaluated.

The questionnaire was distributed through Egyptian association of orthodontist's partner to 200 members, 105 have responded and agreed to participate in this research. Each clinician was required to answer section 1 and the go to section 2 .

Table 1: The cephalometric digital tracing results for case (1)

\begin{tabular}{|c|c|c|c|c|c|c|}
\hline Variable & $1^{\text {st }}$ tracing & $2^{\text {nd }}$ tracing & $3^{\text {rd }}$ tracing & $4^{\text {th }}$ tracing & Mean & Egyptian norms \\
\hline SNA & 87.5 & 87 & 89 & 88.3 & 87.7 & $83^{\circ}[ \pm 3]$ \\
\hline SNB & 90 & 89 & 88 & 88.4 & 88.8 & $80^{\circ}[ \pm 3]$ \\
\hline ANB & -1 & -1.6 & -1 & -1.1 & -1.1 & $3^{\circ}[ \pm 2]$ \\
\hline SN/PP & 10 & 10.7 & 8.3 & 9 & 9.5 & $8.5^{\circ}[ \pm 4]$ \\
\hline SN/Man & 22.8 & 19.2 & 24 & 22.5 & 22.1 & $33^{\circ}[ \pm 5]$ \\
\hline U1/PP & 132.4 & 131 & 128 & 130 & 127 & $114^{\circ}[ \pm 4]$ \\
\hline L1/Man & 89 & 94.4 & 90.5 & 91 & 91.2 & $95^{\circ}[ \pm 6]$ \\
\hline U1/L1 & 110 & 111 & 125 & 111 & 114.2 & $126^{\circ}[ \pm 7]$ \\
\hline Nasolabial angle $\left[\mathrm{Cm}-\mathrm{Sn}-\mathrm{Ls}^{\circ}\right]$ & 106 & 104 & 103 & 107 & 105 & $104^{\circ}[ \pm 10]$ \\
\hline Facial convexity angle [G-Sn-Pg] & 175 & 176 & 174.2 & 175 & 175 & $166^{\circ}[ \pm 5]$ \\
\hline
\end{tabular}

Table 2: The cephalometric digital tracing results for case (2).

\begin{tabular}{|c|c|c|c|c|c|c|}
\hline Variable & $1^{\text {st t }}$ tracing & $2^{\text {nd }}$ tracing & $3^{\text {rd }}$ tracing & $4^{\text {th }}$ tracing & Mean & Egyptian norms \\
\hline SNA & 84 & 83 & 82.7 & 83.7 & 83.3 & $83^{\circ}[ \pm 3]$ \\
\hline SNB & 80.2 & 80 & 80.4 & 80.6 & 80.3 & $80^{\circ}[ \pm 3]$ \\
\hline ANB & 4.4 & 4 & 3.1 & 4 & 3.8 & $3^{\circ}[ \pm 2]$ \\
\hline SN/PP & 2.6 & 3.7 & 2.2 & 2.5 & 2.3 & $8.5^{\circ}[ \pm 4]$ \\
\hline SN/Man & 26 & 26.5 & 26 & 26.3 & 26.2 & $33^{\circ}[ \pm 5]$ \\
\hline U1/PP & 113 & 113.7 & 113 & 115 & 113.6 & $114^{\circ}[ \pm 4]$ \\
\hline L1/Man & 98 & 97 & 96 & 99 & 97.5 & $95^{\circ}[ \pm 6]$ \\
\hline U1/L1 & 127 & 125.4 & 126 & 121 & 124.8 & $126^{\circ}[ \pm 7]$ \\
\hline Nasolabial angle $\left[\mathrm{Cm}-\mathrm{Sn}-\mathrm{Ls}^{\circ}\right]$ & 100 & 99 & 104 & 100.4 & 100.8 & $104^{\circ}[ \pm 10]$ \\
\hline Facial convexity angle [G-Sn-Pg] & 171 & 170 & 127.4 & 170 & 171 & $166^{\circ}[ \pm 5]$ \\
\hline
\end{tabular}

Table 3: The cephalometric digital tracing results for case (3)

\begin{tabular}{|c|c|c|c|c|c|c|}
\hline Variable & $1^{\text {st }}$ tracing & $2^{\text {nd }}$ tracing & $3^{\text {rd }}$ tracing & $4^{\text {th }}$ tracing & Mean & Egyptian norms \\
\hline SNA & 91 & 90 & 90.3 & 90.5 & 90.4 & $83^{\circ}[ \pm 3]$ \\
\hline SNB & 81 & 81 & 80.3 & 80.9 & 80.8 & $80^{\circ}[ \pm 3]$ \\
\hline ANB & 10 & 9 & 10 & 9.7 & 9.6 & $3^{\circ}[ \pm 2]$ \\
\hline SN/PP & 3.5 & 3 & 3.4 & 3.2 & 3.3 & $8.5^{\circ}[ \pm 4]$ \\
\hline SN/Man & 26.3 & 26 & 26.5 & 26.1 & 26.2 & $33^{\circ}[ \pm 5]$ \\
\hline U1/PP & 119.8 & 120 & 121 & 120.1 & 120 & $114^{\circ}[ \pm 4]$ \\
\hline L1/Man & 104.7 & 117 & 103 & 105 & 104 & $95^{\circ}[ \pm 6]$ \\
\hline U1/L1 & 112 & 111 & 112.9 & 111.5 & 111.8 & $126^{\circ}[ \pm 7]$ \\
\hline Nasolabial angle $\left[\mathrm{Cm}-\mathrm{Sn}-\mathrm{Ls}^{\circ}\right]$ & 84 & 83 & 86 & 85 & 84.5 & $104^{\circ}[ \pm 10]$ \\
\hline Facial convexity angle [G-Sn-Pg] & 162 & 163 & 163 & 162 & 162.5 & $166^{\circ}[ \pm 5]$ \\
\hline
\end{tabular}


Table 4: The cephalometric digital tracing results for case (4)

\begin{tabular}{|c|c|c|c|c|c|c|}
\hline Variable & $1^{\text {st }}$ tracing & $2^{\text {nd }}$ tracing & $3^{\text {rd }}$ tracing & $4^{\text {th }}$ tracing & Mean & Egyptian norms \\
\hline SNA & 77.6 & 79 & 75.4 & 78.06 & 78.3 & $83^{\circ}[ \pm 3]$ \\
\hline SNB & 75.5 & 78 & 78 & 76.19 & 76.9 & $80^{\circ}[ \pm 3]$ \\
\hline ANB & 2.1 & 1 & 0.4 & 2 & 1.4 & $3^{\circ}[ \pm 2]$ \\
\hline SN/PP & 12 & 11 & 11.5 & 11.7 & 11.5 & $8.5^{\circ}[ \pm 4]$ \\
\hline SN/Man & 42 & 41.5 & 40 & 41.6 & 41.3 & $33^{\circ}[ \pm 5]$ \\
\hline U1/PP & 125 & 123 & 120.5 & 126 & 123.6 & $114^{\circ}[ \pm 4]$ \\
\hline L1/Man & 104 & 99 & 101 & 101 & 101.3 & $95^{\circ}[ \pm 6]$ \\
\hline U1/L1 & 106 & 107 & 110 & 103 & 106.5 & $126^{\circ}[ \pm 7]$ \\
\hline Nasolabial angle $\left[\mathrm{Cm}-\mathrm{Sn}-\mathrm{Ls}^{\circ}\right]$ & 109 & 112 & 115 & 114 & 112 & $104^{\circ}[ \pm 10]$ \\
\hline Facial convexity angle [G-Sn-Pg] & 165 & 164.7 & 163.7 & 162 & 163.8 & $166^{\circ}[ \pm 5]$ \\
\hline
\end{tabular}

Table 5: The cephalometric digital tracing results for case (5)

\begin{tabular}{|c|c|c|c|c|c|c|}
\hline Variable & $1^{\text {st }}$ tracing & $2^{\text {nd }}$ tracing & $3^{\text {rd }}$ tracing & $4^{\text {th }}$ tracing & Mean & Egyptian norms \\
\hline SNA & 75 & 80 & 74.9 & 75.2 & 76.3 & $83^{\circ}[ \pm 3]$ \\
\hline SNB & 69 & 73 & 68.8 & 69.3 & 70 & $80^{\circ}[ \pm 3]$ \\
\hline ANB & 6 & 7 & 6.1 & 5.9 & 6.3 & $3^{\circ}[ \pm 2]$ \\
\hline SN/PP & 6 & 7.6 & 7.6 & 6.7 & 6.9 & $8.5^{\circ}[ \pm 4]$ \\
\hline SN/Man & 38.5 & 39 & 40.4 & 40.1 & 39.5 & $33^{\circ}[ \pm 5]$ \\
\hline U1/PP & 115 & 114.7 & 113 & 115 & 114.5 & $114^{\circ}[ \pm 4]$ \\
\hline L1/Man & 97.5 & 95 & 93 & 93.1 & 94.65 & $95^{\circ}[ \pm 6]$ \\
\hline U1/L1 & 115 & 119 & 118 & 118.2 & 117.6 & $126^{\circ}[ \pm 7]$ \\
\hline Nasolabial angle $\left[\mathrm{Cm}-\mathrm{Sn}-\mathrm{Ls}^{\circ}\right]$ & 95 & 94 & 100 & 96 & 96.2 & $104^{\circ}[ \pm 10]$ \\
\hline Facial convexity angle [G-Sn-Pg] & 164.3 & 163 & 164 & 164.3 & 163.9 & $166^{\circ}[ \pm 5]$ \\
\hline
\end{tabular}

Table 6: The cephalometric digital tracing results for case (6)

\begin{tabular}{|c|c|c|c|c|c|c|}
\hline Variable & $1^{\text {st }}$ tracing & $2^{\text {nd }}$ tracing & $3^{\text {rd }}$ tracing & $4^{\text {th }}$ tracing & Mean & Egyptian norms \\
\hline SNA & 79 & 79 & 78.8 & 78.5 & 78.8 & $83^{\circ}[ \pm 3]$ \\
\hline SNB & 80 & 80.2 & 80 & 80.3 & 80.1 & $80^{\circ}[ \pm 3]$ \\
\hline ANB & -1 & -1.2 & -1.2 & -1.8 & -1.3 & $3^{\circ}[ \pm 2]$ \\
\hline SN/PP & 11.5 & 11 & 11.8 & 11.5 & 11.45 & $8.5^{\circ}[ \pm 4]$ \\
\hline SN/Man & 39.7 & 39.5 & 40 & 40 & 39.8 & $33^{\circ}[ \pm 5]$ \\
\hline U1/PP & 109 & 108 & 109 & 108.7 & 108.8 & $114^{\circ}[ \pm 4]$ \\
\hline L1/Man & 89 & 88.4 & 88 & 88 & 88.35 & $95^{\circ}[ \pm 6]$ \\
\hline U1/L1 & 135 & 135.4 & 135.6 & 134.5 & 135.1 & $126^{\circ}[ \pm 7]$ \\
\hline Nasolabial angle $\left[\mathrm{Cm}-\mathrm{Sn}-\mathrm{Ls}^{\circ}\right]$ & 93 & 93.2 & 93.8 & 93.5 & 93.4 & $104^{\circ}[ \pm 10]$ \\
\hline Facial convexity angle [G-Sn-Pg] & 177 & 176 & 175 & 177.2 & 176.3 & $166^{\circ}[ \pm 5]$ \\
\hline
\end{tabular}

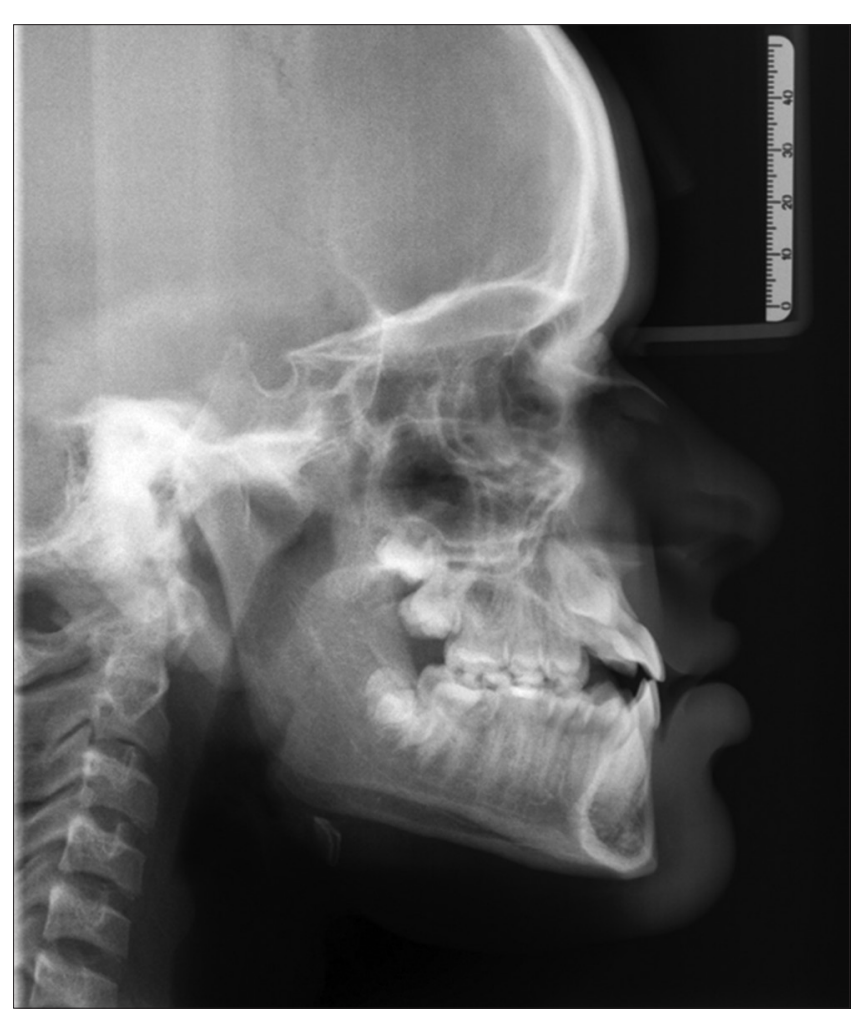

Figure 1: Case (1) skeletal Class III with horizontal facial pattern.

Accordingly, the recruited sample was 105 orthodontists divided equally into three groups. Group 1: Included orthodontists with more than 10 years of experience, Group 2: Included orthodontists with 5-10 years of experience, and

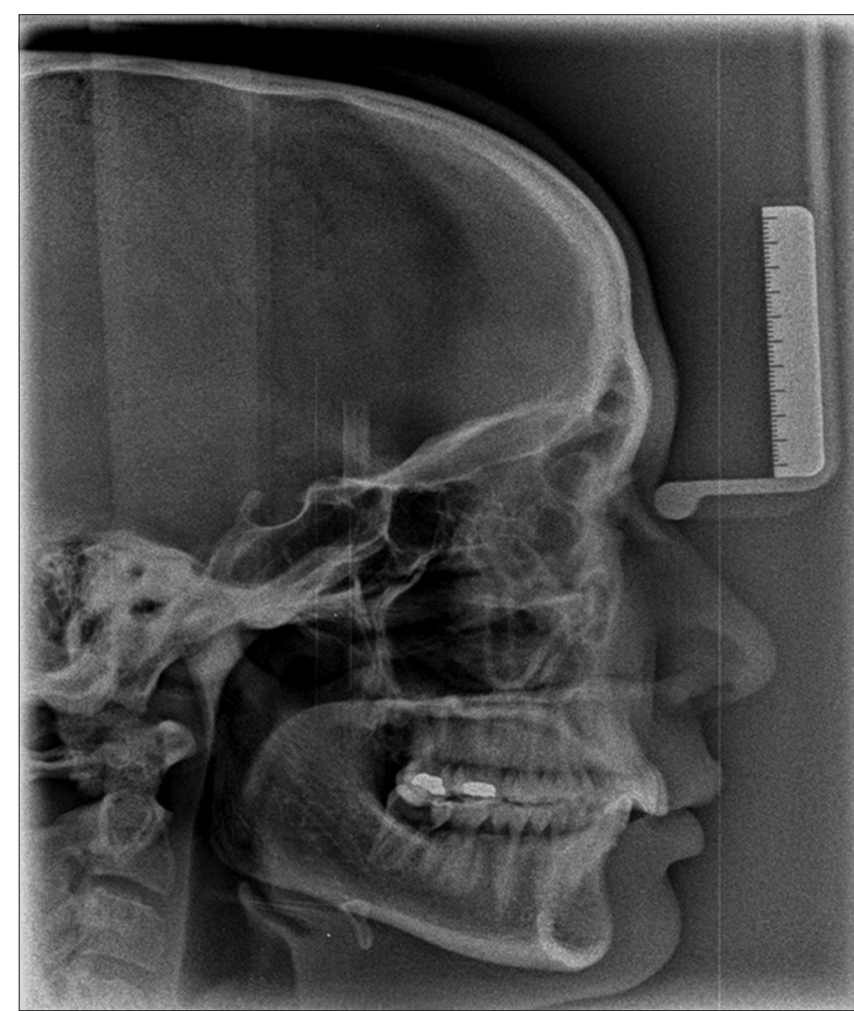

Figure 2: Case (2) skeletal Class I with horizontal facial pattern.

Group 3: Included orthodontists with $<5$ years of experience.

All orthodontists were asked about 8 items for each case skeletal class, facial pattern, upper incisor 


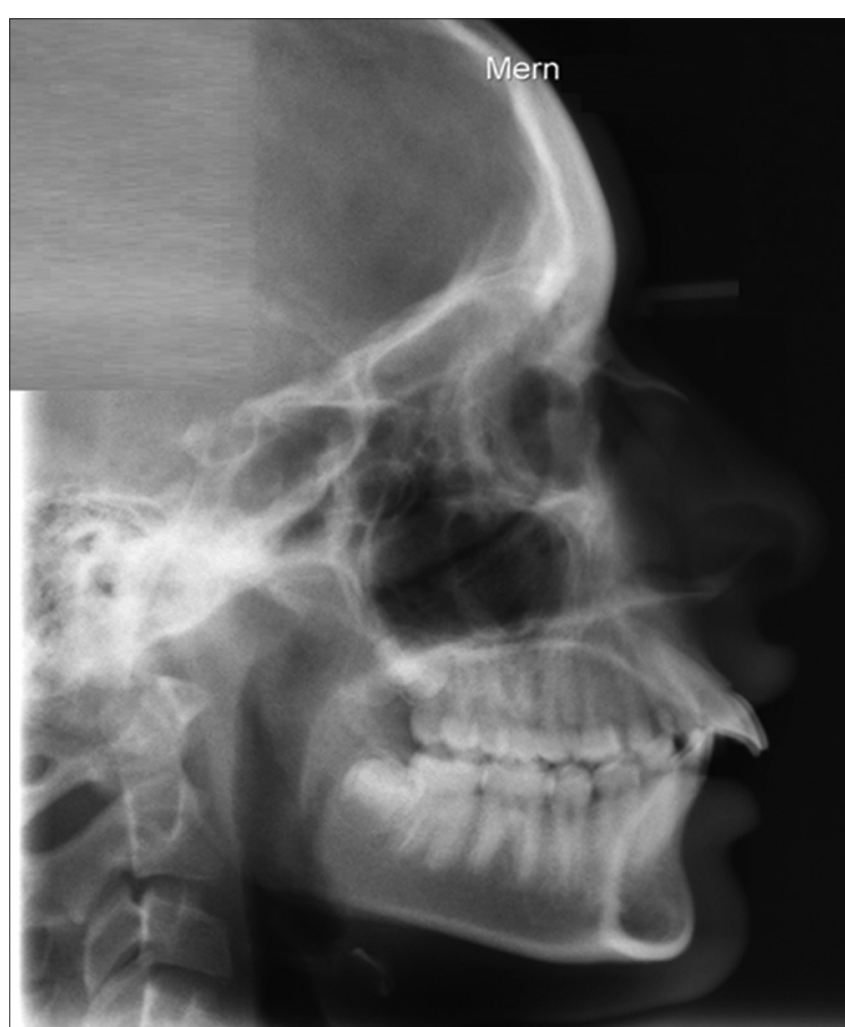

Figure 3: Case (3) skeletal Class II with horizontal facial pattern.

inclination, lower incisor inclination, AP maxillary position, AP mandibular position, nasolabial angle, and facial profile. Then, the results were sent to first author through e-mail and then collected together for statistical analysis.

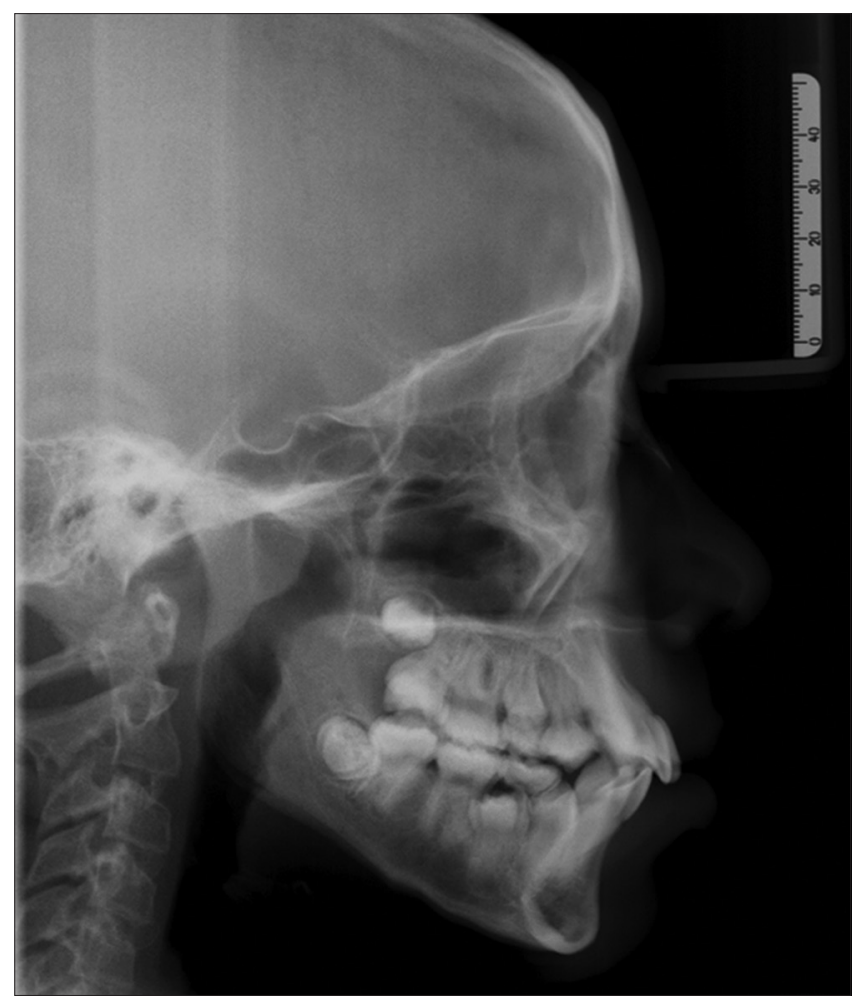

Figure 4: Case (4) skeletal Class I with vertical facial pattern.

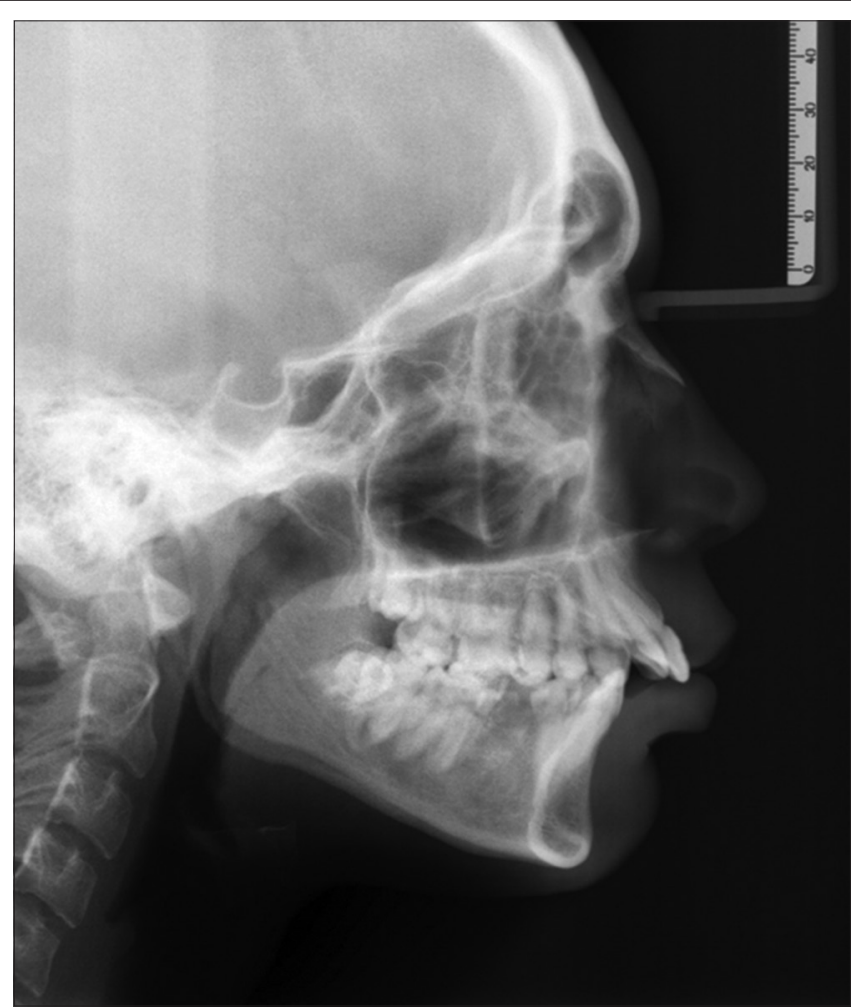

Figure 5: Case (5) skeletal Class // with vertical facial pattern.

\section{Sample size}

The power analysis used in this study was based on the results of Niousha et al. [2]. Sample size of 35 orthodontists per group was needed to detect a

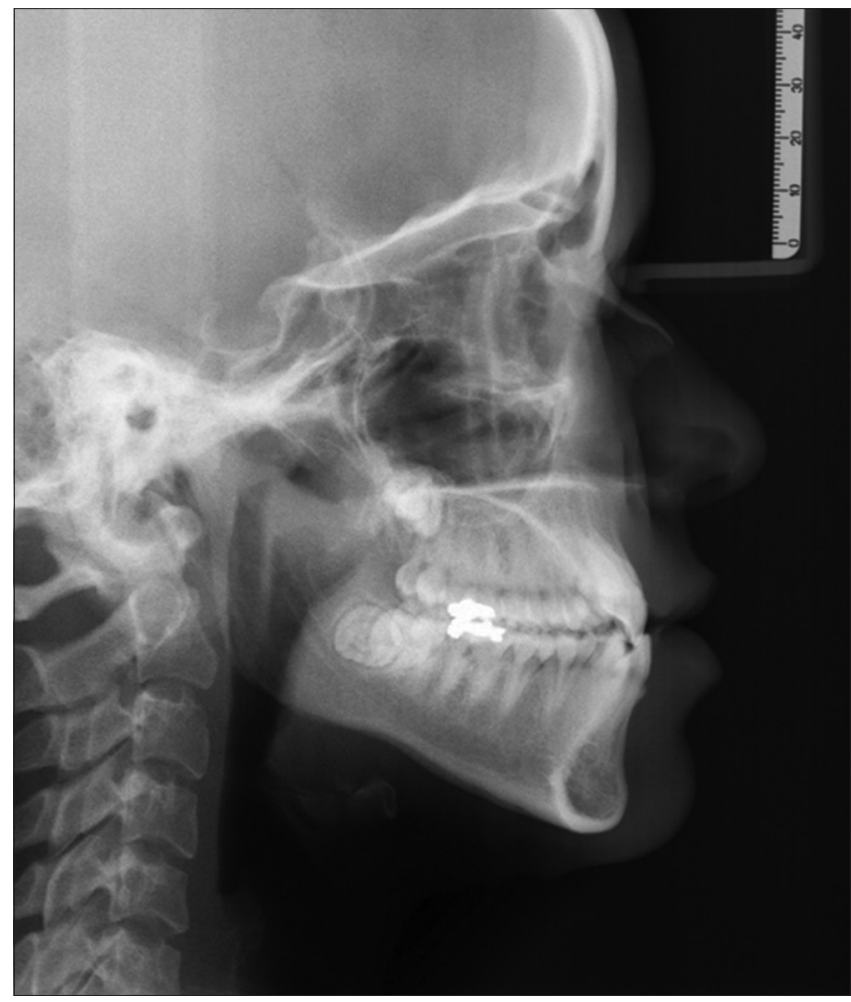

Figure 6: Case (6) skeletal Class III with vertical facial pattern. 
difference of $20 \%$ between any of the three different experience level groups ( $<5$ years, $5-10$ years, and more than 10 years of experience) and the golden reference assuming a 0.05 significance level and $80 \%$ power.

\section{Statistical analysis}

Qualitative data were presented as frequencies and percentages. Quantitative data were presented as median, range, mean, and standard deviation values. Chi-square test or Fisher's exact test (when more than $20 \%$ of the cells have expected frequencies $<5$ ) was used for comparisons regarding qualitative data. Kruskal-Wallis test was used to compare between scores of orthodontists with different years of experience. Dunn's test was used for pair-wise comparisons when Kruskal-Wallis test is significant. Mann-Whitney U-test was used to compare between scores of female and male orthodontists.

The significance level was set at $p \leq 0.05$. Statistical analysis was performed with IBM $®$ SPSS $®$ Statistics Version 20 for Windows.

\section{Results}

\section{Demographic data}

The present study was conducted on 105 orthodontists; 69 males $(65.7 \%)$ and 36 females (34.3\%). Thirty-six orthodontists $(34.3 \%)$ teach in a graduate program while 69 orthodontists $(65.7 \%)$ were private practices.

\section{Comparison between tracing behaviors of orthodontists with different years of experience}

There was a statistically significant difference between percentages of traced cases in the three groups $(p<0.001$, effect size $=0.430)$. Orthodontists with more than 10 years of experience reported that the percentage of cases traced in their practices was between 0 and $25 \%$. On the other hand, the orthodontists with 5-10 years of experience reported that $50 \%$ of the cases were traced in their practices while orthodontists with $<5$ years of experience showed higher prevalence of tracing $(100 \%)$ of the cases (Table 7$)$.

There was a statistically significant difference between percentages of requesting pre-treatment lateral cephalometric radiographs in the three groups $(p<$ 0.001 , effect size $=0.395$ ). Orthodontists with more than 10 years of experience reported percentage of requesting pre-treatment lateral cephalometric radiographs between 25 and $50 \%$ of the cases. On the other hand, the orthodontists with 5-10 years of experience reported that $75 \%$ of the cases were requested pre-treatment lateral cephalometric radiographs while orthodontists with $<5$ years of experience showed higher prevalence of requesting pre-treatment lateral cephalometric radiographs $(100 \%)$ of the cases (Table 7$)$.

Orthodontists with more than 10 years of experience reported percentage of requesting post-treatment lateral cephalometric radiographs between 0 and $25 \%$ of the cases. On the other hand, the orthodontists with 5-10 years of experience reported that $50 \%$ of the cases were requested posttreatment lateral cephalometric radiograph while orthodontists with $<5$ years of experience showed higher prevalence of requesting post-treatment lateral cephalometric radiographs between 75 and $100 \%$ of the cases (Table 7).

\section{Comparison between correct tracing of the} six cases by orthodontists with different years of experience

Participants in the present study analyzed six cases with eight points for each case so that the total score was 48 points.

Skeletal class: There was a statistically significant difference between the three groups regarding correct identification of the skeletal class $(p<0.001$, effect size $=0.374)$.

Table 7: Descriptive statistics and results of Fisher's exact test for comparison between tracing behaviors of orthodontists with different years of experience

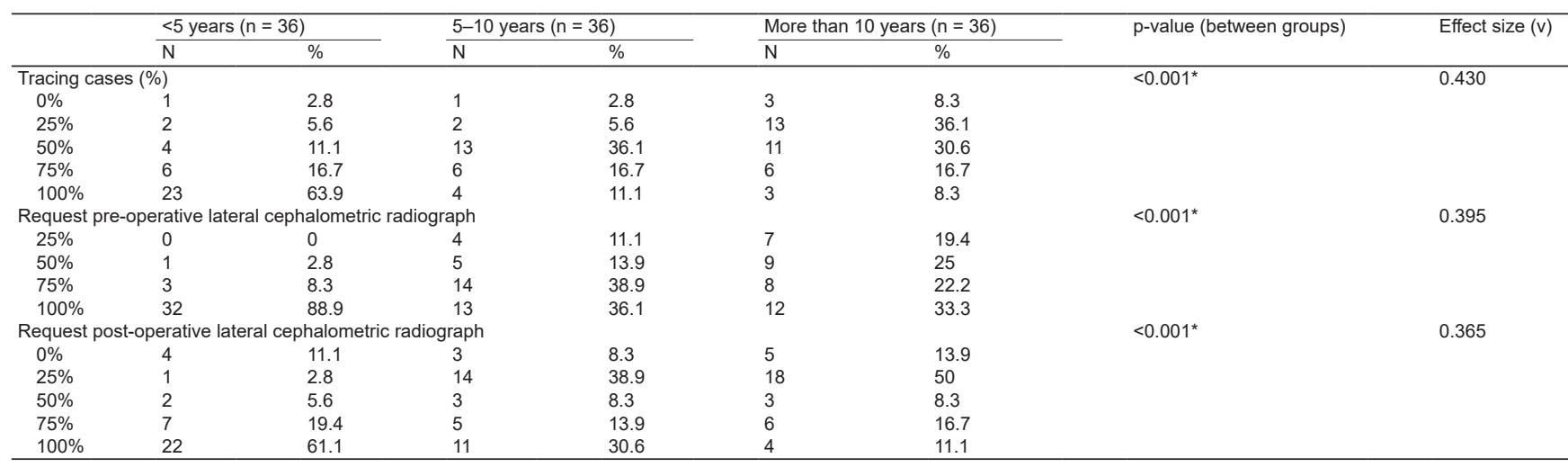


Table 8: Descriptive statistics and results of Chi-square test or Fisher's exact test for comparison between correct tracing of the six cases by orthodontists with different years of experience

\begin{tabular}{|c|c|c|c|c|c|c|c|c|}
\hline \multirow[t]{2}{*}{$\begin{array}{l}\text { Correct tracing } \\
\text { (out of six cases) }\end{array}$} & \multicolumn{2}{|c|}{$\begin{array}{l}<5 \text { years } \\
(n=36)\end{array}$} & \multicolumn{2}{|c|}{$\begin{array}{l}5-10 \text { years } \\
(n=36)\end{array}$} & \multicolumn{2}{|c|}{$\begin{array}{l}\text { More than } 10 \\
\text { years }(n=36)\end{array}$} & \multirow{2}{*}{$\begin{array}{l}\mathrm{p} \text {-value } \\
\text { (between } \\
\text { groups) }\end{array}$} & \multirow[t]{2}{*}{$\begin{array}{l}\text { Effect } \\
\text { size (v) }\end{array}$} \\
\hline & $\mathrm{N}$ & $\%$ & $\mathrm{~N}$ & $\%$ & $\mathrm{~N}$ & $\%$ & & \\
\hline \multicolumn{7}{|l|}{ Skeletal class } & $<0.001^{*}$ & 0.374 \\
\hline $2 / 6$ & 0 & 0 & 2 & 5.6 & 1 & 2.8 & & \\
\hline $3 / 6$ & 4 & 11.1 & 14 & 38.9 & 1 & 2.8 & & \\
\hline $4 / 6$ & 16 & 44.4 & 9 & 25 & 8 & 22.2 & & \\
\hline $5 / 6$ & 14 & 38.9 & 10 & 27.8 & 18 & 50 & & \\
\hline $6 / 6$ & 2 & 5.6 & 1 & 2.8 & 8 & 22.2 & & \\
\hline \multicolumn{3}{|l|}{ Maxillary position } & & & & & 0.089 & 0.288 \\
\hline $0 / 6$ & 2 & 5.6 & 0 & 0 & 0 & 0 & & \\
\hline $1 / 6$ & 3 & 8.3 & 9 & 25 & 2 & 5.6 & & \\
\hline $2 / 6$ & 13 & 36.1 & 13 & 36.1 & 15 & 41.7 & & \\
\hline $3 / 6$ & 15 & 41.7 & 14 & 38.9 & 16 & 44.4 & & \\
\hline $4 / 6$ & 3 & 8.3 & 0 & 0 & 1 & 2.8 & & \\
\hline $6 / 6$ & 0 & 0 & 0 & 0 & 2 & 5.6 & & \\
\hline \multicolumn{3}{|c|}{ Mandibular position } & & & & & 0.370 & 0.234 \\
\hline $1 / 6$ & 2 & 5.6 & 2 & 5.6 & 2 & 5.6 & & \\
\hline $2 / 6$ & 9 & 25 & 12 & 33.3 & 7 & 19.4 & & \\
\hline $3 / 6$ & 12 & 33.3 & 15 & 41.7 & 16 & 44.4 & & \\
\hline $4 / 6$ & 12 & 33.3 & 4 & 11.1 & 6 & 16.7 & & \\
\hline $5 / 6$ & 1 & 2.8 & 3 & 8.3 & 3 & 8.3 & & \\
\hline $6 / 6$ & 0 & 0 & 0 & 0 & 2 & 5.6 & & \\
\hline \multicolumn{3}{|l|}{ Growth pattern } & & & & & $0.002^{*}$ & 0.352 \\
\hline $1 / 6$ & 0 & 0 & 2 & 5.6 & 0 & 0 & & \\
\hline $2 / 6$ & 8 & 22.2 & 8 & 22.2 & 7 & 19.4 & & \\
\hline $3 / 6$ & 9 & 25 & 17 & 47.2 & 8 & 22.2 & & \\
\hline $4 / 6$ & 18 & 50 & 5 & 13.9 & 10 & 27.8 & & \\
\hline $5 / 6$ & 0 & 0 & 3 & 8.3 & 6 & 16.7 & & \\
\hline $6 / 6$ & 1 & 2.8 & 1 & 2.8 & 5 & 13.9 & & \\
\hline \multicolumn{5}{|c|}{ Upper incisor inclination } & & & 0.169 & 0.226 \\
\hline $2 / 6$ & 2 & 5.6 & 0 & 0 & 3 & 8.3 & & \\
\hline $3 / 6$ & 18 & 50 & 11 & 30.6 & 9 & 25 & & \\
\hline $4 / 6$ & 11 & 30.6 & 18 & 50 & 14 & 38.9 & & \\
\hline $5 / 6$ & 5 & 13.9 & 5 & 13.9 & 8 & 22.2 & & \\
\hline $6 / 6$ & 0 & 0 & 2 & 5.6 & 2 & 5.6 & & \\
\hline \multicolumn{5}{|c|}{ Lower incisor inclination } & & & 0.118 & 0.286 \\
\hline $1 / 6$ & 3 & 8.3 & 0 & 0 & 0 & 0 & & \\
\hline $2 / 6$ & 4 & 11.1 & 3 & 8.3 & 2 & 5.6 & & \\
\hline $3 / 6$ & 10 & 27.8 & 11 & 30.6 & 12 & 33.3 & & \\
\hline $4 / 6$ & 16 & 44.4 & 20 & 55.6 & 12 & 33.3 & & \\
\hline $5 / 6$ & 3 & 8.3 & 2 & 5.6 & 8 & 22.2 & & \\
\hline $6 / 6$ & 0 & 0 & 0 & 0 & 2 & 5.6 & & \\
\hline \multicolumn{3}{|l|}{ Nasolabial angle } & & & & & 0.257 & 0.255 \\
\hline $1 / 6$ & 3 & 8.3 & 1 & 2.8 & 1 & 2.8 & & \\
\hline $2 / 6$ & 11 & 30.6 & 6 & 16.7 & 12 & 33.3 & & \\
\hline $3 / 6$ & 12 & 33.3 & 16 & 44.4 & 10 & 27.8 & & \\
\hline $4 / 6$ & 9 & 25 & 8 & 22.2 & 10 & 27.8 & & \\
\hline $5 / 6$ & 1 & 2.8 & 5 & 13.9 & 1 & 2.8 & & \\
\hline $6 / 6$ & 0 & 0 & 0 & 0 & 2 & 5.6 & & \\
\hline \multicolumn{3}{|l|}{ Facial profile } & & & & & 0.102 & 0.265 \\
\hline $0 / 6$ & 13 & 36.1 & 13 & 36.1 & 10 & 27.8 & & \\
\hline $1 / 6$ & 9 & 25 & 12 & 33.3 & 7 & 19.4 & & \\
\hline $2 / 6$ & 7 & 19.4 & 9 & 25 & 11 & 30.6 & & \\
\hline $3 / 6$ & 4 & 11.1 & 0 & 0 & 6 & 16.7 & & \\
\hline $4 / 6$ & 3 & 8.3 & 2 & 5.6 & 0 & 0 & & \\
\hline $6 / 6$ & 0 & 0 & 0 & 0 & 2 & 5.6 & & \\
\hline
\end{tabular}

Orthodontists with more than 10 years of experience showed higher prevalence of correct eyeball tracing results of $5 / 6$ and 6/6 cases?.

- Maxillary position: There was no statistically significant difference between the three groups regarding correct eyeball tracing results of maxillary position ( $p=0.089$, effect size $=0.288$ ).

- $\quad$ Mandibular position: There was no statistically significant difference between the three groups regarding correct eyeball tracing results of mandibular position ( $p=0.370$, effect size $=0.234$ ).

- Growth pattern: There was a statistically significant difference between the three groups regarding correct identification of the growth pattern $(p=0.002$, effect size $=0.352)$. Orthodontists with more than 10 years of experience showed higher prevalence of correct $t$ eyeball tracing results of $5 / 6$ and $6 / 6$ cases?.

- Upper incisor inclination: There was no statistically significant difference between the three groups regarding correct tracing of upper incisor inclination $(p=0.169$, effect size $=0.226$ ).

Lower incisor inclination: There was no statistically significant difference between the three groups regarding correct eyeball tracing results of lower incisor inclination $(p=0.118$, effect size $=0.286$ ) .

- Nasolabial angle: There was no statistically significant difference between the three groups regarding correct eyeball tracing results of nasolabial angle $(p=0.257$, effect size $=0.255)$. Facial profile: There was no statistically significant difference between the three groups regarding correct eyeball tracing results of facial profile $(p=0.102$, effect size $=0.265)$.

\section{Total score}

There was a statistically significant difference between the three groups regarding total score of score of conformity of eyeball tracing with digital tracing results $(p=0.004$, effect size $=0.085)$. Pair-wise comparisons revealed that orthodontists with more than 10 years of experience showed the statistically significantly highest median score. There was no statistically significant difference between orthodontists with experience $<5$ years and those with 5-10 years of experience; both showed statistically significantly lower median scores.

\section{Comparison between tracing behaviors of female and male orthodontists}

There was no statistically significant difference between percentages of in practice traced cases by female and male orthodontists ( $p=0.663$, effect size $=0.155)$ (Table 9).

Table 9: Descriptive statistics and results of Fisher's exact test for comparison between tracing behaviors of female and male orthodontists

\begin{tabular}{|c|c|c|c|c|c|c|}
\hline & \multicolumn{2}{|c|}{ Females $(n=36)$} & \multicolumn{2}{|c|}{ Males $(n=69)$} & \multirow{2}{*}{$\begin{array}{l}\text { p-value (between } \\
\text { groups) }\end{array}$} & \multirow{2}{*}{$\begin{array}{l}\text { Effect size } \\
\text { (v) }\end{array}$} \\
\hline & $\mathrm{n}$ & $\%$ & $\mathrm{n}$ & $\%$ & & \\
\hline \multicolumn{5}{|c|}{ Tracing cases (\%) } & 0.663 & 0.155 \\
\hline $0 \%$ & 1 & 2.7 & 4 & 5.6 & & \\
\hline $25 \%$ & 13 & 35.1 & 17 & 23.9 & & \\
\hline $50 \%$ & 7 & 18.9 & 20 & 28.2 & & \\
\hline $75 \%$ & 9 & 24.3 & 19 & 26.8 & & \\
\hline $100 \%$ & 7 & 18.9 & 11 & 15.5 & & \\
\hline \multicolumn{5}{|c|}{$\begin{array}{l}\text { Request pre-operative lateral cephalometric } \\
\text { radiograph }\end{array}$} & 0.301 & 0.184 \\
\hline $25 \%$ & 3 & 8.1 & 8 & 11.3 & & \\
\hline $50 \%$ & 6 & 16.2 & 9 & 12.7 & & \\
\hline $75 \%$ & 5 & 13.5 & 20 & 28.2 & & \\
\hline $100 \%$ & 23 & 62.2 & 34 & 47.9 & & \\
\hline \multicolumn{5}{|c|}{$\begin{array}{l}\text { Request post-operative lateral cephalometric } \\
\text { radiograph }\end{array}$} & 0.502 & 0.178 \\
\hline $0 \%$ & 2 & 5.4 & 10 & 14.1 & & \\
\hline $25 \%$ & 10 & 27 & 23 & 32.4 & & \\
\hline $50 \%$ & 4 & 10.8 & 4 & 5.6 & & \\
\hline $75 \%$ & 6 & 16.2 & 12 & 16.9 & & \\
\hline $100 \%$ & 15 & 40.5 & 22 & 31 & & \\
\hline
\end{tabular}


There was no statistically significant difference between percentages of requesting pretreatment lateral cephalometric radiographs by female and male orthodontists $(p=0.301$, effect size $=0.184)$ (Table 9).

There was no statistically significant difference between percentages of requesting posttreatment lateral cephalometric radiographs by female and male orthodontists $(p=0.502$, effect size $=0.178)$ (Table 9).

\section{Comparison between correct tracing scores of the six cases by orthodontists with different years of experience}

There was no statistically significant difference between females and males regarding dentoskeletal and soft-tissue parameter that evaluated in this study (Table 10).

Table 10: Descriptive statistics and results of Chi-square test or Fisher's exact test for comparison between correct tracing of the six cases by female and male orthodontists

\begin{tabular}{|c|c|c|c|c|c|c|}
\hline \multirow{2}{*}{$\begin{array}{l}\text { Correct tracing } \\
\text { (Out of } 6 \text { cases) }\end{array}$} & \multicolumn{2}{|c|}{ Females $(n=36)$} & \multicolumn{2}{|c|}{ Males $(n=69)$} & \multirow{2}{*}{$\begin{array}{l}\text { p-value (between } \\
\text { groups) }\end{array}$} & \multirow{2}{*}{$\begin{array}{l}\text { Effect } \\
\text { Size (v) }\end{array}$} \\
\hline & $\mathrm{N}$ & $\%$ & $\mathrm{~N}$ & $\%$ & & \\
\hline \multicolumn{5}{|l|}{ Skeletal class } & 0.331 & 0.203 \\
\hline $2 / 6$ & 1 & 2.7 & 2 & 2.8 & & \\
\hline $3 / 6$ & 4 & 10.8 & 15 & 21.1 & & \\
\hline $4 / 6$ & 9 & 24.3 & 24 & 33.8 & & \\
\hline $5 / 6$ & 19 & 51.4 & 23 & 32.4 & & \\
\hline $6 / 6$ & 4 & 10.8 & 7 & 9.9 & & \\
\hline \multicolumn{5}{|l|}{ Maxillary position } & 0.841 & 0.127 \\
\hline $0 / 6$ & 1 & 2.7 & 1 & 1.4 & & \\
\hline $1 / 6$ & 3 & 8.1 & 11 & 15.5 & & \\
\hline $2 / 6$ & 15 & 40.5 & 26 & 36.6 & & \\
\hline $3 / 6$ & 16 & 43.2 & 29 & 40.8 & & \\
\hline $4 / 6$ & 1 & 2.7 & 3 & 4.2 & & \\
\hline $6 / 6$ & 1 & 2.7 & 1 & 1.4 & & \\
\hline \multicolumn{5}{|c|}{ Mandibular position } & 0.789 & 0.150 \\
\hline $1 / 6$ & 1 & 2.7 & 5 & 7 & & \\
\hline $2 / 6$ & 12 & 32.4 & 16 & 22.5 & & \\
\hline $3 / 6$ & 13 & 35.1 & 30 & 42.3 & & \\
\hline $4 / 6$ & 8 & 21.6 & 14 & 19.7 & & \\
\hline $5 / 6$ & 2 & 5.4 & 5 & 7 & & \\
\hline $6 / 6$ & 1 & 2.7 & 1 & 1.4 & & \\
\hline \multicolumn{5}{|l|}{ Growth pattern } & 0.387 & 0.210 \\
\hline $1 / 6$ & 1 & 2.7 & 1 & 1.4 & & \\
\hline $2 / 6$ & 10 & 27 & 13 & 18.3 & & \\
\hline $3 / 6$ & 9 & 24.3 & 25 & 35.2 & & \\
\hline $4 / 6$ & 9 & 24.3 & 24 & 33.8 & & \\
\hline $5 / 6$ & 5 & 13.5 & 4 & 5.6 & & \\
\hline $6 / 6$ & 3 & 8.1 & 4 & 5.6 & & \\
\hline \multicolumn{5}{|c|}{ Upper incisor inclination } & 0.646 & 0.155 \\
\hline $2 / 6$ & 2 & 5.4 & 3 & 4.2 & & \\
\hline $3 / 6$ & 12 & 32.4 & 26 & 36.6 & & \\
\hline $4 / 6$ & 18 & 48.6 & 25 & 35.2 & & \\
\hline $5 / 6$ & 4 & 10.8 & 14 & 19.7 & & \\
\hline $6 / 6$ & 1 & 2.7 & 3 & 4.2 & & \\
\hline \multicolumn{5}{|c|}{ Lower incisor inclination } & 0.807 & 0.146 \\
\hline $1 / 6$ & 2 & 5.4 & 1 & 1.4 & & \\
\hline $2 / 6$ & 2 & 5.4 & 7 & 9.9 & & \\
\hline $3 / 6$ & 11 & 29.7 & 22 & 31 & & \\
\hline $4 / 6$ & 17 & 45.9 & 31 & 43.7 & & \\
\hline $5 / 6$ & 4 & 10.8 & 9 & 12.7 & & \\
\hline $6 / 6$ & 1 & 2.7 & 1 & 1.4 & & \\
\hline \multicolumn{5}{|l|}{ Nasolabial angle } & 0.184 & 0.250 \\
\hline $1 / 6$ & 3 & 8.1 & 2 & 2.8 & & \\
\hline $2 / 6$ & 9 & 24.3 & 20 & 28.2 & & \\
\hline $3 / 6$ & 16 & 43.2 & 22 & 31 & & \\
\hline $4 / 6$ & 8 & 21.6 & 19 & 26.8 & & \\
\hline $5 / 6$ & 0 & 0 & 7 & 9.9 & & \\
\hline $6 / 6$ & 1 & 2.7 & 1 & 1.4 & & \\
\hline \multicolumn{5}{|l|}{ Facial profile } & 0.333 & 0.228 \\
\hline $0 / 6$ & 13 & 35.1 & 23 & 32.4 & & \\
\hline $1 / 6$ & 13 & 35.1 & 15 & 21.1 & & \\
\hline $2 / 6$ & 7 & 18.9 & 20 & 28.2 & & \\
\hline $3 / 6$ & 3 & 8.1 & 7 & 9.9 & & \\
\hline $4 / 6$ & 0 & 0 & 5 & 7 & & \\
\hline $6 / 6$ & 1 & 2.7 & 1 & 1.4 & & \\
\hline
\end{tabular}

\section{Discussion}

Diagnosis and treatment planning carry great significance in orthodontic treatment. Various factors affect them and they vary considerably from one orthodontist to another, among these factors are the clinician's trait and experience. One of the major problems in orthodontic diagnosis and assessment of orthodontic treatment need is that an orthodontic anomaly is not a disease with a series of well-recognized symptoms it is a variation from the norm in which treatment is based on the evaluation of certain dental characteristics in an otherwise healthy patient [6].

The importance of clinician's trait becomes evident when different orthodontists are given a patient scenario of a border line case. Some clinicians choose to extract while others option for non-extraction [2].

The concerns about treatment quality and reducing treatment costs has been an important subject of current scientific discussion in orthodontics over the last decade and a half [7].

Examinations concerning the prevalence or necessity of malocclusion treatment often have contradictory results. This is partly due to differing attempts and methods of examination but also to variations between the examiners [8].

With the advent of cephalometric head films, various analyses were developed in an attempt to qualitative and quantitative esthetic facial profiles.

The cephalometric analysis has been used as the standard method because of the ease of procuring, measuring, and comparing (superimposition) hard tissue structures. These perceived advantages of cephalometric analysis have led to heavy reliance on cephalometry in all aspects of orthodontic treatment [9].

The purpose of this study was to evaluate the influence of orthodontist's gender and level of experience on the perception of dentoskeletal parameters. Six cases for evaluation were included in this study which were distributed among 105 orthodontists with different level of experience to assist their perception to different dentoskeletal parameters as stated in the objective for current study.

The cases were utilized from the records of patients in the Orthodontic Department of the Faculty of Oral and Dental Medicine at Future University in Egypt. This study was approved by the ethical committee of the faculty of dental medicine, Future University in Egypt.

The variables used in this study were commonly used cephalometric variables for orthodontic diagnosis, treatment planning, and evaluation of treatment results. Steiner's, Wit's, Tweed's, McNamara, Rakosi, and 
Jarabaks analyses are commonly used for orthognathic surgical planning, hard tissue, dental variables, and soft-tissue variables.

The accuracy of cephalometric analysis is important in the diagnosis of malocclusion and for treatment planning. Rapid advances in computer technology have led to increasing use of digital systems in cephalometry. The most important criteria for using mechanical or digital method are that it should be accurate, precise, and must show a high rate of reproducibility in both tracing and analysis [11]. Studies comparing digital and manual cephalometric analysis methods revealed that computer-assisted cephalometric analysis yielded comparable results to the manual method [7], [11].

Digital cephalograms obtained by various digitization processes or digital radiography. The clinician needs only to identify the landmarks and let the program calculate the cephalometric measurements [12].

There are many errors with the traditional method arise from radiographic acquisition, landmark identification, measurement, and observer experience [13], [14], [15]. A previous study revealed that computer-aided cephalometric analysis did not introduce more measurement errors when localization of the landmarks was determined by hand [16]. A more recent study concluded that the differences between all skeletal and dental measurements derived from the landmarks on original cephalometric radiographs and those identified on their digitized counterparts were statistically significant but clinically acceptable [12].

Landmark identification is as important as the tracing method itself because interoperator error has in general been found to be greater than intraoperator error as indicated by Sayinsu et al. [17]. To avoid such errors, measurements were carried out by single orthodontist (main investigator EE). The identification process was performed with low luminosity and under the same conditions, as recommended by Houston [18].

The electronic survey was developed using SurveyMonkey®. The advantages of online surveys are that being faster, cheaper, easy to use, more accurate, more quick, more selective, and more flexible [19].

The present study was conducted on 105 orthodontists; 69 males $(65.7 \%)$ and 36 females (34.3\%). Thirty-six orthodontists (34.3\%) teach in a graduate program while 69 orthodontists $(65.7 \%)$ do not teach in any graduate programs. Exploring the association between the perception of dentoskeletal parameters and experience a clear trend was observed. Orthodontists with more than 10 years of experience showed higher prevalence of correct eyeballs tracing more than orthodontists with less experience hence were the idea of distributing the sample into three groups with different years of experience.

The result of this study indicated that there could be an association between clinician's experience and the perception of dentoskeletal parameters. A few other studies have evaluated the influence of the clinician's experience on extraction treatment decision-making. In all three scenarios, clinicians with more than 15 years of experience choose an extraction treatment option almost twice more often than those with less experience. Baelum et al. [1] have found that orthodontic experience was the only factor that could be correlated with differences in treatment plans by different orthodontists.

Niousha et al. [2] have found that orthodontics with more than 15 years of experience choose an extraction treatment option more frequently than clinician's with $<5$ or 15 years of experience so it is reasonable to assume that orthodontists' past experiences may play a role in their treatment decisions.

The results of this study suggested that gender does not play a role perception of dentoskeletal parameters. In every case, there was no statistically significant difference between females and males regarding correct identification of the skeletal class, growth pattern, lower incisor inclination, maxillary position, upper incisor inclination, mandibular position, nasolabial angle, and facial profile. The same results were achieved when our data were stratified based on gender and experience. Baelum et al. [1] have also shown that gender does not influence treatment decision-making. Niousha et al. [2] in 2017 have found that there is no association between gender and place of education and the decision to extract in the selected borderline cases.

According to the results aforementioned, the experience plays an important role in perception of dentoskeletal parameters on lateral cephalometric radiographs. Orthodontist with more than 10 year of experience showed higher prevalence of perception of dentoskeletal parameters while there was no correlation between clinician's gender in perception of dentoskeletal parameters.

\section{Conclusion}

Orthodontists with more than 10 years of experience showed higher prevalence of perception of dentoskeletal parameters on lateral cephalometric radiographs than the less experienced groups. No association was found between clinician's gender and perception of dentoskeletal parameters.

\section{References}

1. Baelum V, Borchorst E, Buch H, Dømgaard P, Hartig LE. Inter examiner variability in orthodontic treatment decisions for Danish children with 'borderline' treatment need. Eur J Orthod. 2011;34:250-6. https://doi.org/10.1093/ejo/cjq195 


\section{PMid:21252189}

2. Niousha S, Heaton LJ, Burcu B, David L, Roozbeh K, AnneMarie B. Influence of clinicians' experience and gender on extraction decision in orthodontics. Angle Orthod. 2017;87(5):641-50. https://doi.org/10.2319/020117-80.1 PMid:28686094

3. Satravaha S. The significance of the integumentary profile. Am J Orthod. 1987;92(5):422-6. https://doi. org/10.1016/0889-5406(87)90263-0

PMid:3479010

4. Kay EJ, Nuttal $\mathrm{N}$. Clinical decision making $\mathrm{n}$ art or science. Part II making sense of treatment decisions. $\mathrm{Br}$ Dent $\mathrm{J}$. 1995;180:113-7. https://doi.org/10.1038/sj.bdj.4808664

5. Patrick JH, Strough J. Everyday problem solving: Experience, strategies and behavioral intentions. J Adult Dev. 2004;11:9-19. https://doi.org/10.1023/b: jade.0000012523.31728.f7

6. Richmond S, O'Brien KD, Roberts CT, Andrews M. Dentists variation in the determination of orthodontic treatment need. Br J Orthod. 1994;21(1):65-8. https://doi.org/10.1179/ bjo.21.1.65

PMid:8199167

7. Gesch D, Schröder W, Kocher T, Kirbschus A. Examiner differences in the assessment of different malocclusions. J Orofac Orthop. 2006;67(6):404-13. https://doi.org/10.1007/ s00056-006-0627-4

PMid:17124559

8. Gesch D, Bernhardt O, Alte D, et al. Prevalence of signs and symptoms of temporomandibular disorders in an urban and rural German population: Results of a population-based study of health in Pomerania. Quintessence Int. 2004;35(2):143-50. PMid: 15000638

9. Arnett GW, Bergman RT. Facial keys to orthodontic diagnosis and treatment planning. Part I. Am J Orthod Dentofac Orthop. 1993;103(4):299-312. https://doi. org/10.1016/0889-5406(93)70010-I PMid:8480695

10. Sandler PJ. Reproducibility of cephalometric measurements. $\mathrm{Br}$ J Orthod. 1988;15:105-10. https://doi.org/10.1179/bjo.15.2.105 PMid:3165025
11. Santoro M, Jarjoura K, Cangialosi TJ. Accuracy of digital and analogue cephalometric measurements assesses with the sandwich technique. Am J Orthod Dentofacial Orthop. 2006,129(3):345-51. https://doi.org/10.1016/j. ajodo.2005.12.010 PMid:16527629

12. Chen YJ, Chen SK, Yao JC, Chang HF. The effects of differences in landmark identification on the cephalometric measurements in traditional versus digitized cephalometry. Angle Orthod. 2004;74:155-61.

PMid: 15132440

13. Björk A, Solow B. Measurement on radiographs. J Dent Res. 1962;41:672-83.

PMid:13971297

14. Houston WJ, Maher RE, McElroy D, Sherriff M. Sources of error in measurements from cephalometric radiographs. Eur J Orthod. 1986;8:149-51. https://doi.org/10.1093/ejo/8.3.149 PMid:3464438

15. Forsyth DB, Shaw WC, Richmond S. Digital imaging of cephalometric radiology. Part I: Advantages and limitation of digital imaging. Angle Orthod. 1996;66(1):37-42.

PMid:8678344

16. Gravely JF, Benzies PM. The clinical significance of tracing error in cephalometry. Br J Orthod. 1974;1(3):95-101. https:// doi.org/10.1179/bjo.1.3.95 PMid:4525738

17. Sayinsu K, Isik F, Trakyali G, Arun T. An evaluation of the errors in cephalometric measurements on scanned cephalometric images and conventional tracings. Eur J Orthod. 2007;29(1):1058. https://doi.org/10.1093/ejo/cjl065

PMid: 17290023

18. Houston WJ. The analysis of errors in orthodontic measurements. Am J Orthod. 1983;83(5):382-90. https://doi. org/10.1016/0002-9416(83)90322-6

PMid:6573846

19. Alwin DF, Krosnick JA. The reliability of survey attitude measurement. The influence of question and respondent attributes. Sociol Methods Res. 1991;20:139-81. https://doi. org/10.1177/0049124191020001005

\section{Appendix}

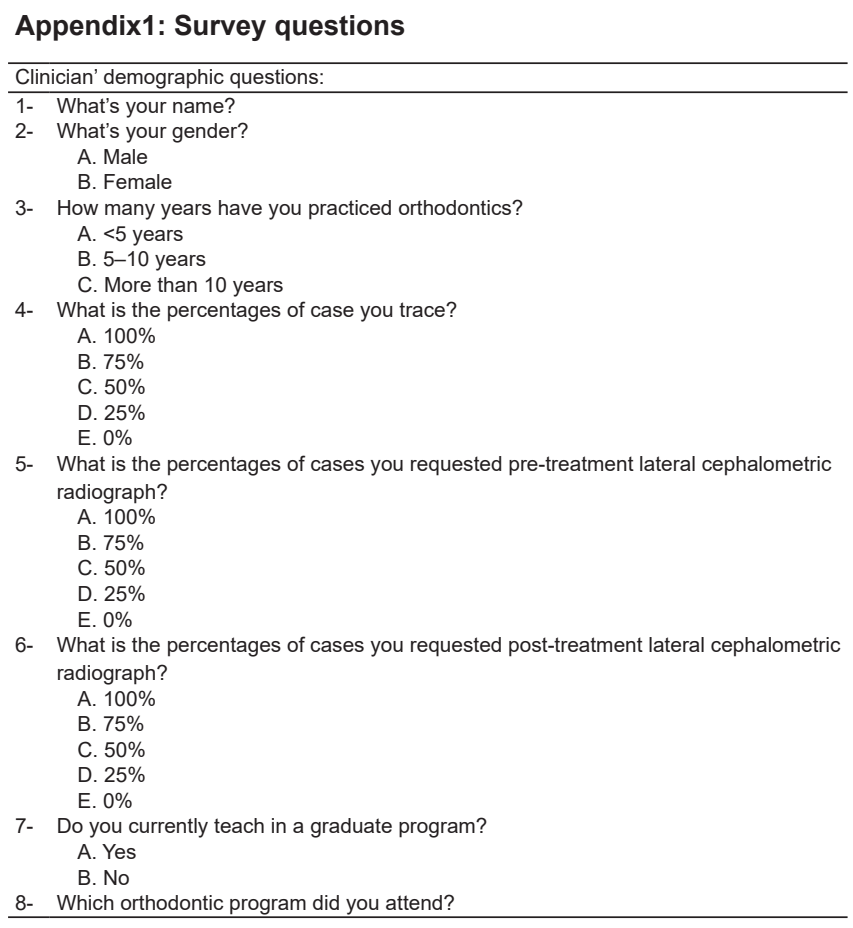

